\title{
Prevalence of chronic kidney disease after preeclampsia
}

\author{
Veronica Agatha Lopes van Balen ${ }^{1}$ (1) Julia Jeltje Spaan ${ }^{1} \cdot$ Tom Cornelis $^{2}$ • \\ Marc Erich August Spaanderman ${ }^{1}$
}

Received: 25 May 2016/ Accepted: 27 July 2016/Published online: 5 August 2016

(c) The Author(s) 2016. This article is published with open access at Springerlink.com

\begin{abstract}
Background Preeclampsia (PE), an endothelial disease that affects kidney function during pregnancy, is correlated to an increased future risk of cardiovascular and chronic kidney disease. The Kidney Disease Improving Global Outcomes (KDIGO) 2012 guideline emphasizes the combined role of glomerular filtration rate (GFR) and albuminuria in determining the frequency of monitoring of kidney function.

Objectives In this study we evaluated the prevalence of CKD in women with a history of PE. We investigated how many seemingly healthy women required monitoring of kidney function according to the KDIGO guideline.

Methods We included 775 primiparous women with a history of PE. They were at least 4 months postpartum, and had no pre-existing hypertension, diabetes or kidney disease. We estimated GFR by the CKD-Epidemiology equation and urinary albumin loss by albumin creatinine ratio in a 24-h urine collection.

Results Most women, 669 (86.3\%), had a normal GFR and absent albuminuria. Based on the KDIGO guideline, $13.7 \%$ would require at least yearly monitoring of kidney
\end{abstract}

Electronic supplementary material The online version of this article (doi:10.1007/s40620-016-0342-1) contains supplementary material, which is available to authorized users.

Veronica Agatha Lopes van Balen

v.lopesvanbalen@gmail.com

1 Department of Obstetrics and Gynecology, Maastricht University Medical Center, PO Box 5800, 6202 AZ Maastricht, The Netherlands

2 Division of Nephrology, Department of Internal Medicine, Maastricht University Medical Center, Maastricht, The Netherlands function. Only $1.4 \%$ were classified to be at high risk for kidney function deterioration.

Conclusion Monitoring of kidney function seems relevant for about one in seven women with a history of PE, mainly due to albuminuria. Albuminuria should be evaluated postpartum to identify those women that need further monitoring of kidney function.

Keywords Kidney function · Preeclampsia - Postpartum · KDIGO criteria

\section{Introduction}

Preeclampsia (PE), an endothelial disease characterized by hypertension and commonly coinciding with proteinuria, is the most common cause of maternal death in the Netherlands. Maternal death due to preeclampsia occurs in 3.5 of 100,000 live births, accounting for $39 \%$ of total maternal deaths [1]. PE affects kidney function during pregnancy and also increases the risk of future chronic hypertension, chronic kidney disease (CKD) and cardiovascular disease [2-9]. PE is associated with a fourfold increased risk of developing end-stage renal disease (ESRD) within 10 years after pregnancy [3]. Having more than one preeclamptic pregnancy, a low-birthweight offspring or a preterm delivery increases this risk even further [3].

Glomerular endotheliosis observed during a preeclamptic pregnancy may be fully reversible if it does not reach the level of glomerular scarring [10]. The reversibility is obviously dependent on cessation of the cause of endothelial injury. In the case of PE, this is effectuated by termination of pregnancy and delivery of the placenta. It can take months before the fibrinous and granular material deposits in the glomerulus fully disappear [11]. 
Data on follow-up of kidney function of these women are scarce. Some case-control and cohort studies suggest that most women with a history of PE do maintain good kidney function after PE [12-14]. However, there remains a group of women that will have decreased glomerular filtration rate (GFR) and/or persistent urinary protein loss $[2,5]$.

The existence of moderately increased albuminuria, at any glomerular filtration rate, is considered an independent risk factor for the development of cardiovascular disease [15-17]. The Kidney Disease Improving Global Outcomes (KDIGO) 2012 criteria emphasize the independent role of GFR and albuminuria in assessing the prognosis of CKD. The guideline provides us with comprehensive evidencebased indications for monitoring of patients with CKD. In this study, we determined the prevalence of CKD in a large cohort of women with a history of PE. We aimed to establish how many of these seemingly healthy former patients would qualify for further monitoring of kidney function according to the KDIGO guideline.

\section{Methods}

In the Maastricht University Medical Center (MUMC) a postpartum cardiovascular screening is offered to women with a history of PE. The aim of this screening is to determine underlying disorders that might have contributed to the development of PE and to advise them on future pregnancies and cardiovascular implications. All women with a history of a vascular complicated pregnancy (either maternal hypertensive or fetal growth restricted) were offered a postpartum cardiovascular screening after delivery in our hospital, and some were also referred from other hospitals in the country, between 1996 and 2014. For our analysis, to improve the homogeneity of the studied population, we selected only women with PE (gestational hypertensive with de novo proteinuria) [18] who entered our program. We excluded multiparous and women who were evaluated less than 4 months after delivery. We also excluded women diagnosed with hypertension, diabetes mellitus or kidney disease prior to the complicated pregnancy. All measurements were performed in our assessment unit at the Maastricht University Medical Center under controlled circumstances.

\section{Measurements}

Measurements during the postpartum cardiovascular screening were performed in standardized environmental conditions. Blood pressure was recorded for a period of $30 \mathrm{~min}$ at 3-min intervals using a semiautomatic oscillometric device in half-sitting position. Median values of nine subsequent recordings were used for analysis. An extensive (family) history was taken. Urine analysis was done to determine albumin loss on a urine sample collected over $24 \mathrm{~h}$. Albuminuria was measured by immunochemical analysis. We categorized women into normal to mild albuminuria $<3 \mathrm{mg} / \mathrm{mmol}$, moderately increased albuminuria $3-30 \mathrm{mg} / \mathrm{mmol}$, and severely increased albuminuria $>30 \mathrm{mg} / \mathrm{mmol}$ [19]. We also collected blood samples for serum creatinine, which was obtained by the Jaffe method until 2012 and thereafter through an enzymatic method. All three measurements were performed with Cobas 8000 modular analyzer series (Roche Diagnostics, Basel, Switzerland).

We estimated GFR by the CKD-Epidemiology (CKDEPI) equation in $\mathrm{ml} / \mathrm{min} / 1.73 \mathrm{~m}^{2}$. Both formulas are dependent on serum creatinine and used for females and Caucasians. When serum creatinine was below or equal to $0.7 \mathrm{mg} / \mathrm{dl}$, we used $144 \times(\text { serum creatinine } / 0.7)^{-0.329} \times$ $0.993^{\text {age }}$. When serum creatinine was above $0.7 \mathrm{mg} / \mathrm{dl}$ we used $144 \times(\text { serum creatinine } / 0.7)^{-1.209} \times 0.993^{\text {age }}$. Albuminuria was quantified by the albumin creatinine ratio (ACR).

\section{KDIGO}

The KDIGO guideline provides a table with recommendations on frequency of monitoring of kidney function based on the risk of progression of CKD, and another table on monitoring by a general clinician or referral to a nephrologist. We combined both tables into one overview table (Table 1) [19]. In this table the yellow color indicates a preferred frequency of monitoring once a year, whereas orange indicates monitoring twice a year, light red three times a year, and dark red four times a year or more. Referral for monitoring of kidney function by more specialized personnel, preferably a nephrologist, is indicated for severely increased albuminuria $($ ACR $>30)$ or decreased GFR $(<30)$, according to the KDIGO guideline, as is shown.

The KDIGO table on cardiovascular mortality is based on a meta-analysis [20] of 105,872 individuals, extracted from 14 different studies (see Table S1). To calculate the adjusted relative risk of cardiovascular mortality for our study group we classified all women based on this table.

\section{Statistical analysis}

All analyses were performed using SPSS version 21.0, property of IBM (SPSS, Chicago, IL, USA) and supplied by Maastricht University. Data were expressed as group means and standard deviation or medians and interquartile ranges (IQR). A p value $<0.05$ was considered to indicate a statistically significant difference. To analyze the differences between groups with normally distributed data we 
Table 1 KDIGO table on frequency of monitoring showing the prevalence of chronic kidney disease in primiparous women with a history of preeclampsia

\begin{tabular}{|c|c|c|c|}
\hline$N=775$ & $A C R<3$ & ACR 3-30 & $A C R>30$ \\
\hline GFR $>89$ & 573 (73.9\%) & $83(10.7 \%)$ & $9(1.2 \%)$ \\
\hline GFR 60-89 & $96(12.4 \%)$ & $12(1.6 \%)$ & $1(0.1 \%)$ \\
\hline GFR 45-59 & Monitoring & & $1(0.1 \%)$ \\
\hline GFR 30-44 & & & \\
\hline GFR 15-29 & & Referral & \\
\hline GFR $<15$ & & & \\
\hline
\end{tabular}

Frequency of monitoring based on the risk of progression of CKD depending on ACR and GFR [19]. Once-yearly monitoring is represented by yellow, twice-yearly by orange, three times per year by light red, and four times per year by dark red. GFR is expressed in $\mathrm{ml} /$ $\mathrm{min} / 1.73 \mathrm{~m}^{2}$ and ACR in $\mathrm{mg} / \mathrm{mmol}$. Referral indicates the need for referral to more specialized care, preferably by a nephrologist

KDIGO Kidney Disease Improving Global Outcomes, GFR glomerular filtration rate, $A C R$ albumin creatinine ratio

used the unpaired $\mathrm{t}$ test. For non-normal distributed data, we used the Mann-Whitney U test. Dichotomous data were analyzed by Chi square test. We corrected for months postpartum, age, gestational onset of hypertensive complication and gestational age at delivery where appropriate.

\section{Results}

Women with a history of PE, at least 4 months postpartum, were selected after exclusion of those that were multiparous or had pre-existing hypertension, diabetes mellitus and/or kidney disease (Fig. 1). Data required for categorization of kidney function were incomplete in $3.1 \%$ of cases, leaving 775 complete cases.

Table 2 shows the characteristics of our study group. Women were on average 10 months postpartum, normotensive, had an early onset $\mathrm{PE}(<34$ weeks gestation) and gave birth prematurely. The baseline values were equal to those of women excluded due to missing urinary analyses. Women with persistent moderate to severely increased albuminuria (ACR $>3 \mathrm{mg} / \mathrm{mmol}$ ) were younger, gave birth at an earlier gestational age to smaller children and had higher blood pressure compared to women with normal to mildly increased albuminuria (ACR $<3 \mathrm{mg} / \mathrm{mmol}$ ). They had an average amount of protein loss of $196 \mathrm{mg} / 24 \mathrm{~h}$ [IQR 154-237] and used significantly more antihypertensive drugs, in particular renin-angiotensin system (RAS) blocking drugs. Based on the KDIGO guideline on the frequency of monitoring (Table 1), for $106(13.7 \%)$ women with a history of PE, monitoring of kidney function was indicated once yearly (yellow category). This was mostly due to their having moderate-to-severely increased ACR with a normal GFR (>90) (11.9\%). Only $1.8 \%$ had a decreased GFR $(<90)$ and moderate-to-severely increased ACR. In addition, $11(1.4 \%)$ women resulted as needing more specialized care, preferably by a nephrologist, based on severely increased albuminuria (ACR $>30 \mathrm{mg} / \mathrm{mmol}$ ). Table 3 details the postpartum renal albumin loss categorized by time interval. Moderate to increased albumin loss was most frequent within the first months after delivery and was less prominent in women who were more than $1 \frac{1}{2}$ years postpartum. Women who were 2 years postpartum had an increased ACR in $6 \%$ of cases. Table S1 (supplementary appendix) presents the KDIGO table on calculated cardiovascular mortality risk for our study group. It shows that about $25 \%$ of women had a relative risk of 0.9 of cardiovascular mortality, $22 \%$ a 1.3 relative risk, and $10 \%$ a 1.5 relative risk. Some women $(7.6 \%)$ even had a 2.3 increased risk. The median adjusted relative risk of cardiovascular mortality was $1.25 \pm 0.6$ (not shown in Table S1). The increased cardiovascular mortality risk was mostly based on increased albumin loss as opposed to a reduced GFR.

\section{Discussion}

Our study indicates that, based on the KDIGO monitoring guideline, about one in seven women after PE should have at least yearly monitoring of their kidney function, this being mainly due to a moderately increased albuminuria. Only $1.4 \%$ were classified as at high risk for kidney function deterioration and in need of referral to specialist care, preferably a nephrologist. About $6 \%$ of women had an increased ACR 2 years postpartum.

$\mathrm{PE}$ can manifest as a combination of maternal and fetal factors. It can be considered as more than one disease with major differences between near-term mild PE and PE that is associated with low birthweight and preterm delivery [21]. Our data indicate that mainly persistent albuminuria would classify $13.7 \%$ women after PE as having CKD. The prevalence of CKD in the general adult, and usually older, population is approximately $10 \%$ [22]. Women with a history of PE are younger and have a four to fivefold increased risk of ESRD compared to women with no history of PE [3]. Having more than one preeclamptic pregnancy, a low-birthweight offspring or a preterm delivery increases the risk of CKD even further [3]. 


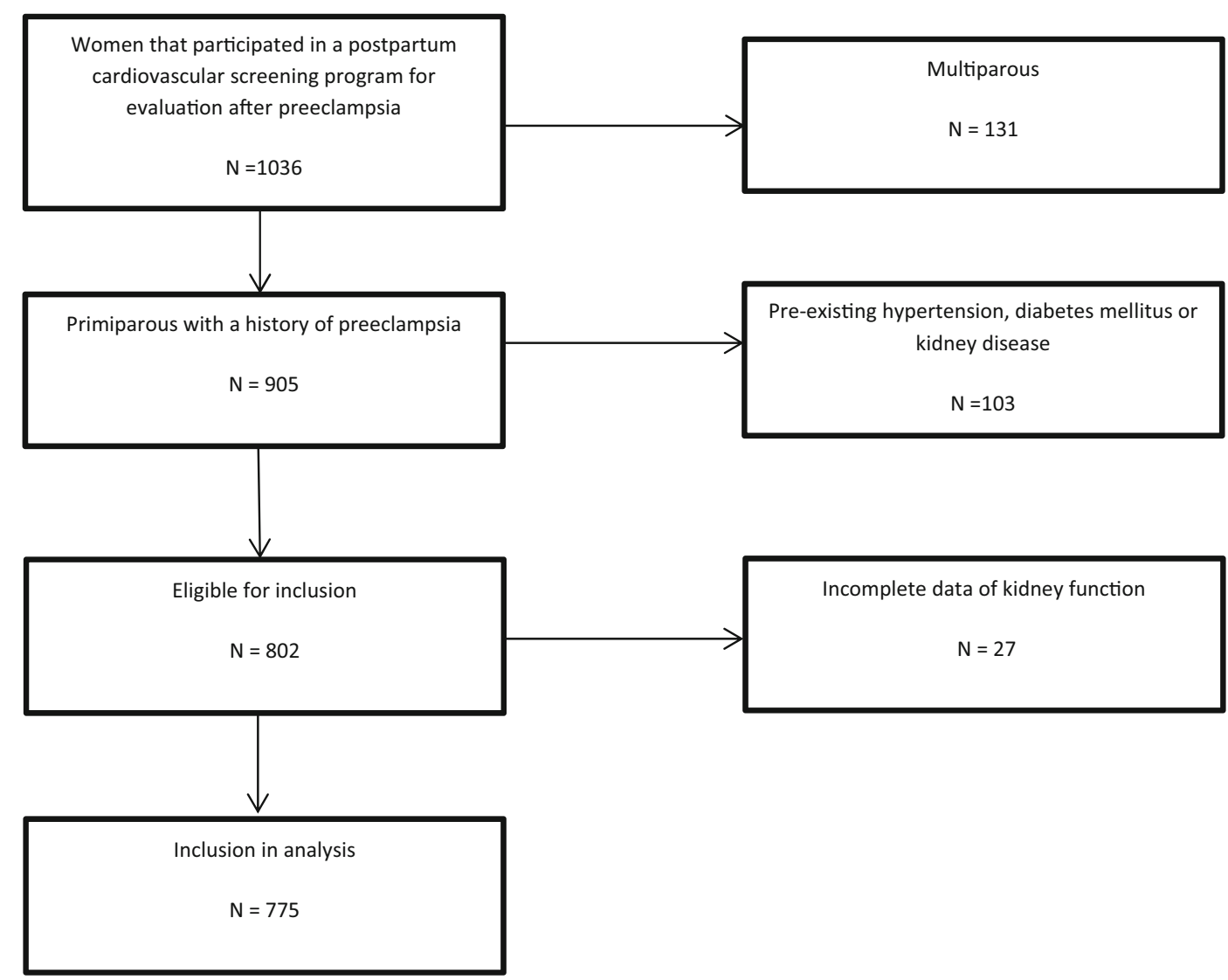

Fig. 1 Flowchart

The KDIGO guideline is written for the general population and indicates that kidney disease persisting for more than 4 months is considered to be chronic, irrespective of the etiology. This provides us with guidance on how often we should follow women with a history of PE after delivery to ensure that albuminuria will regress over time and to clarify which women should be further investigated for underlying kidney disease. The persistence of albuminuria is considered to be due to either an undiagnosed pre-existing kidney disease, or a higher susceptibility of these women to develop CKD, or irreversible kidney damage during the preeclamptic pregnancy. Though kidney disease is known in some cases to be entirely reversible, either spontaneously or with treatment, it is prudent to ascertain whether this actually happens [23].

The prevalence of albuminuria in our study population is lower compared to other data that mention $42 \%$ after 3-5 years postpartum and 20-30\% after 7 years postpartum $[5,12,24,25]$. On the contrary, some other studies conducted at a later postpartum interval show no increased persistent (micro)albuminuria [2,14]. We did, however, exclude women with known conditions that affect kidney function. It could be that, due to the homogeneity of the disease of PE, kidney function is affected differently, depending on the severity of the disease, but also recovers at a different time rate, possibly depending on the woman's general health. Another contributing factor could be the method used for the acquisition of data on albuminuria as some studies use 24-h and others 8-h collection or a single urine sample.

We would also like to address the notion that the risk of cardiovascular mortality is increased in women with a history of PE. As the risk of cardiovascular disease is also increased in women with CKD [26], we could hypothesize that women with a history of PE and CKD have an even more increased risk of cardiovascular disease. Appropriate treatment aimed at preventing progression of cardiovascular disease is important since blood pressure control, lifestyle advice and exercise can slow down or even reverse progression of both CVD and CKD [21]. Appropriate blood pressure regulation seems particularly relevant for this group, as in our study women with persistent albuminuria had a higher blood pressure compared to those with normal to mildly increased albuminuria. They did already use significantly more antihypertensive medication, in particular RAS blocking drugs. Blood pressure regulation, as part of cardiovascular risk management, improves the prognosis of CKD [27]. Albuminuria screening seems not only effective but also cost-effective, when taking possible treatment with 
Table 2 Characteristics of all primiparous women with a history of preeclampsia and stratified by albumin creatinine ratio (ACR)

\begin{tabular}{|c|c|c|c|c|}
\hline & $\begin{array}{l}\text { All participants } \\
\mathrm{n}=775\end{array}$ & $\begin{array}{l}\mathrm{ACR}<3 \mathrm{mg} / \mathrm{mmol} \\
\mathrm{n}=669\end{array}$ & $\begin{array}{l}\mathrm{ACR} \geq 3 \mathrm{mg} / \mathrm{mmol} \\
\mathrm{N}=106\end{array}$ & $\mathrm{p}$ value \\
\hline Age (years) & $31 \pm 4.2$ & $31 \pm 4.1$ & $30 \pm 4.5^{*}$ & $<0.01$ \\
\hline Months postpartum & $10[6-18]$ & $12[7-19]$ & $10[5-10]$ & 0.945 \\
\hline BMI $\left(\mathrm{kg} / \mathrm{m}^{2}\right)$ & $25.0 \pm 4.8$ & $25 \pm 4.6$ & $25 \pm 5.2$ & 0.872 \\
\hline Smoking (\%) & $109(14 \%)$ & $94(14 \%)$ & $15(14 \%)$ & 0.978 \\
\hline Gestational age at diagnosis (weeks) & $31^{+5} \pm 4^{+1}$ & $31^{+6} \pm 4^{+1}$ & $30^{+6} \pm 4^{+0} *$ & 0.049 \\
\hline Gestational age at delivery (weeks) & $33^{+3} \pm 3^{+5}$ & $33^{+4} \pm 3^{+5}$ & $32^{+5} \pm 3^{+6} *$ & 0.012 \\
\hline Birth weight (g) & $1856[1188-2447]$ & $1890[1230-2450]$ & $1657[980-2266]^{*}$ & 0.010 \\
\hline Birth weight percentile & $20[9-40]$ & 20 [9-45] & $18[6-36]^{*}$ & 0.037 \\
\hline Systolic blood pressure $(\mathrm{mmHg})^{\dagger}$ & $116 \pm 13$ & $116 \pm 12$ & $120 \pm 12 *$ & $<0.01$ \\
\hline Diastolic blood pressure $(\mathrm{mmHg})^{\dagger}$ & $72 \pm 9$ & $71 \pm 9$ & $75 \pm 10^{*}$ & $<0.01$ \\
\hline Glomerular filtration rate $\left(\mathrm{ml} / \mathrm{min} / 1.73 \mathrm{~m}^{2}\right)^{\dagger}$ & $105 \pm 15$ & $105 \pm 15$ & $107 \pm 16$ & 0.471 \\
\hline Antihypertensive drugs & $66(8.5 \%)$ & $52(7.7 \%)$ & $14(13.2 \%)^{*}$ & 0.031 \\
\hline ACR mg/mmol & $0.9[0.5-1.9]$ & $0.8[0.5-1.3]$ & $5[4-10]$ & \\
\hline RAS blocking drugs (\%) & $26(3.4 \%)$ & $16(2.4 \%)$ & $10(9.4 \%) *$ & $<0.01$ \\
\hline
\end{tabular}

Data were are expressed as group means and standard deviation or median and interquartile range

$B M I$ body mass index, $R A S$ renin-angiotensin system. For other abbreviations, see previous tables

$* \mathrm{p}<0.05$

+ Adjusted for months postpartum

${ }^{\dagger}$ Adjusted for gestational age at delivery and age

Table 3 Albuminuria after preeclampsia categorized by time interval

\begin{tabular}{lll}
\hline Months postpartum & $>3 \mathrm{ACR} \mathrm{mg} / \mathrm{mmol} \mathrm{n} /$ total $(\%)$ & Adjusted odds \\
\hline $4-5$ & $51 / 150(25 \%)$ & Ref \\
$6-11$ & $33 / 248(12 \%)$ & $0.43(\mathrm{CI} 0.26-0.71)^{*}$ \\
$12-17$ & $13 / 96(12 \%)$ & 0.44 (CI $0.23-0.87)^{*}$ \\
$18-23$ & $1 / 58(2 \%)$ & 0.06 (CI $0.00-0.46)^{*}$ \\
$>24$ & $8 / 117(6 \%)$ & 0.25 (CI $0.11-0.57)^{*}$ \\
\hline
\end{tabular}

Odds ratio represents the risk of persistent albuminuria $(>3 \mathrm{ACR})$ at a certain time period postpartum compared with to the reference group (4-6 months postpartum), corrected for gestational age at delivery and age. An asterisk represents a $\mathrm{p}$ value less than $<0.05$ angiotensin-converting-enzyme (ACE) inhibitors into account, as it reduces the risk of both CKD and cardiovascular disease [28]. Interestingly, screening of albuminuria in a high-risk population is found to correlate with the occurrence of future renal replacement therapy [29]. This makes screening for albuminuria in a population of women with a history of PE even more plausible. From a practical point of view, we would advise evaluation of kidney function, for women with a history of PE, at the regular 6-weeks postpartum control. Women who then have microalbuminuria could be seen 1 year thereafter by their general physician for follow-up of kidney function. If a cardiovascular screening should be available the inclusion of a test for microalbuminuria would be advisable.

There are some shortcomings of our study that limit extrapolation to other populations. First of all, even though the KDIGO offers a guideline with evidence-based decisions that provides the possibility to take both GFR and ACR into account when evaluating kidney function, it is difficult to extrapolate outcomes and the need for frequency of follow-up for young and otherwise healthy women from the CKD tables of KDIGO. It is possible that these women will have very different relative risk changes and rates of progression than corresponding individuals with other renal diseases like diabetes or primary glomerular disease. The KDIGO guideline does not define patients by etiology. Secondly, it may be that our population does not completely represent the general formerly preeclamptic group of women as our population primarily consists of women with early onset PE. Early onset disease may cause an overestimation of the prevalence of CKD due to the severity of the disease and its impact on the 
endothelium. On the other hand, the results might underestimate the prevalence of CKD after PE due to the exclusion of comorbidities known to be related to kidney dysfunction, such as diabetes mellitus, pre-existing hypertension and known kidney disease. The cross-sectional nature of this study is also an inevitable shortcoming, as is the lack of a control group. Because not all women with a history of PE that delivered in our hospital partook in the screening and because women were referred from other hospitals we are not able to fully generalize to the overall PE population. A future longitudinal study is needed to ascertain changes over time, preferably including data on pre-pregnancy GFR or albuminuria values to determine a possible pre-existing kidney condition.

The key strengths of this study are the relatively long postpartum period, when compared to a regular checkup at 6 weeks after delivery and the large study population. Recent studies indicate that CKD-EPI gives the best estimate of GFR when compared to the gold standard of $\mathrm{I}^{125}$ iothalamate [30]. It has the highest accuracy in women when compared to both the MDRD and Cockcroft-Gault formula [31] and the use of CKD-EPI has, to date, not been reported in women with a history of PE.

\section{Conclusion}

Monitoring of kidney function seems relevant for about one in seven formerly preeclamptic women, mainly due to albuminuria. Albuminuria should be evaluated postpartum to identify those women that need further monitoring of kidney function.

\section{Compliance with ethical standards}

Conflict of interest The authors declare that they have no conflict of interest.

Ethical approval All procedures performed in studies involving human participants were in accordance with the ethical standards of the institutional and/or national research committee and with the 1964 Helsinki declaration and its later amendments or comparable ethical standards.

Informed consent For this type of study formal consent is not required.

Funding There was no support/funding for the making of this manuscript.

Open Access This article is distributed under the terms of the Creative Commons Attribution 4.0 International License (http://crea tivecommons.org/licenses/by/4.0/), which permits unrestricted use, distribution, and reproduction in any medium, provided you give appropriate credit to the original author(s) and the source, provide a link to the Creative Commons license, and indicate if changes were made.

\section{References}

1. Schutte JM, Steegers EAP, Schuitemaker NWE, Santema JG, De Boer K, Pel M et al (2010) Rise in maternal mortality in the Netherlands. BJOG 117(4):399-406

2. Spaan JJ, Ekhart T, Spaanderman MEA, Peeters LLH (2009) Remote hemodynamics and renal function in formerly preeclamptic women. Obstet Gynecol 113(4):853-859

3. Vikse BE, Irgens LM, Leivestad T, Skjaerven R, Iversen BM (2008) Preeclampsia and the risk of end-stage renal disease. N Engl J Med 359(8):800-809

4. Vikse BE (2013) Pre-eclampsia and the risk of kidney disease. Lancet 382(9887):104-106

5. McDonald SD, Han Z, Walsh MW, Gerstein HC, Devereaux PJ (2010) Kidney disease after preeclampsia: a systematic review and meta-analysis. Am $J$ Kidney Dis Elsevier Inc 55(6):1026-1039

6. van der Graaf AM, Toering TJ, Faas MM, Lely AT (2012) From preeclampsia to renal disease: a role of angiogenic factors and the renin-angiotensin aldosterone system? Nephrol Dial Transplant 27(Suppl 3):iii51-iii57

7. Vikse BE, Hallan S, Bostad L, Leivestad T, Iversen BM (2010) Previous preeclampsia and risk for progression of biopsy-verified kidney disease to end-stage renal disease. Nephrol Dial Transplant 25(10):3289-3296

8. Bellamy L, Casas J-P, Hingorani AD, Williams DJ (2007) Preeclampsia and risk of cardiovascular disease and cancer in later life: systematic review and meta-analysis. BMJ 335(7627):974

9. Machado S, Figueiredo N, Borges A, Pais MSJ, Freitas L, Moura $P$ et al (2012) Acute kidney injury in pregnancy: a clinical challenge. J Nephrol 25(1):19-30

10. Lafayette RA (2005) The kidney in preeclampsia. Kidney Int 67:1194-1203

11. Hussein W, Lafayette RA (2014) Renal function in normal and disordered pregnancy. Curr Opin Nephrol Hypertens 23(1):46-53

12. Berks D, Steegers EAP, Molas M, Visser W (2009) Resolution of hypertension and proteinuria after preeclampsia. Obstet Gynecol 114(6):1307-1314

13. Lampinen KH, Rönnback M, Groop P-H, Kaaja RJ (2006) Renal and vascular function in women with previous preeclampsia: a comparison of low- and high-degree proteinuria. Kidney Int 70(10): $1818-1822$

14. Sandvik MK, Hallan S, Svarstad E, Vikse BE (2013) Preeclampsia and prevalence of microalbuminuria 10 years later. Clin J Am Soc Nephrol 8(7):1126-1134

15. Chambers JC, Fusi L, Haskard DO, Swiet M De, Page P (2001) Association of maternal endothelial dysfunction with preeclampsia. JAMA 285(12):1607-1612

16. Arnlöv J, Evans JC, Meigs JB, Wang TJ, Fox CS, Levy D et al (2005) Low-grade albuminuria and incidence of cardiovascular disease events in nonhypertensive and nondiabetic individuals: the Framingham Heart Study. Circulation 112(7):969-975

17. Schmieder RE, Schrader J, Zidek W, Tebbe U, Paar WD, Bramlage $P$ et al (2007) Low-grade albuminuria and cardiovascular risk: what is the evidence? Clin Res Cardiol 96(5):247-257

18. Heart N (2000) Report of the National High Blood Pressure Education Program Working Group on High Blood Pressure in Pregnancy. Am J Obstet Gynecol 183(1):s1-s22

19. Levey AS, de Jong PE, Coresh J, El Nahas M, Astor BC, Matsushita K et al (2011) The definition, classification, and prognosis of chronic kidney disease: a KDIGO Controversies Conference report. Kidney Int (Nature Publishing Group) 80(1):17-28

20. van der Velde M, Matsushita K, Coresh J, Astor BC, Woodward M, Levey A et al (2011) Lower estimated glomerular filtration rate and higher albuminuria are associated with all-cause and 
cardiovascular mortality. A collaborative meta-analysis of highrisk population cohorts. Kidney Int (Nature Publishing Group) 79(12):1341-1352

21. Sibai B, Dekker G, Kupferminc M (2005) Pre-eclampsia. Lancet. 365(9461):785-799

22. Levey AS, Coresh J (2012) Chronic kidney disease. Lancet Elsevier Ltd 379(9811):165-180

23. Selvin E, Stevens LA, Manzi J, Eggers P, Lente F Van, Levey AS (2007) Prevalence of chronic kidney disease in the United States. JAMA 298(17):2038-2047

24. Bar J, Kaplan B, Wittenberg C, Erman A, Ben-rafael Z, Hod M (1999) Microalbuminuria after pregnancy complicated by preeclampsia. Nephrol Dial Transpl 14:1129-1132

25. Nisell H, Lintu H, Lunell NO, Mollerstrom G, Pettersson E (1995) Blood pressure and renal function 7 years after pregnancy complicated by hypertension. $\mathrm{Br} \mathrm{J}$ Obstet Gynaecol 102(11):876-881

26. Kurth T, de Jong PE, Cook NR, Buring JE, Ridker PM (2009) Kidney function and risk of cardiovascular disease and mortality in women: a prospective cohort study. BMJ 338:b2392
27. Parati G, Ochoa JE, Bilo G (2012) Blood pressure variability, cardiovascular risk, and risk for renal disease progression. Curr Hypertens Rep 14(5):421-431

28. Boersma C, Gansevoort RT, Pechlivanoglou P, Visser ST, van Toly FFJ, de Jong-van den Berg LTW et al (2010) Screen-andtreat strategies for albuminuria to prevent cardiovascular and renal disease: cost-effectiveness of nationwide and targeted interventions based on analysis of cohort data from the Netherlands. Clin Ther Excerpta Medica Inc 32(6):1103-1121

29. van der Velde M, Halbesma N, de Charro FT, Bakker SJL, de Zeeuw D, de Jong PE et al (2009) Screening for albuminuria identifies individuals at increased renal risk. J Am Soc Nephrol 20(4):852-862

30. Chen J, Muntner P, Hamm LL, Jones DW, Batuman V, Fonseca $\mathrm{V}$ et al (2004) The metabolic syndrome and chronic kidney disease in U.S. adults. Ann Intern Med 140(3):167-174

31. Michels WM, Grootendorst DC, Verduijn M, Elliott EG, Dekker FW, Krediet RT (2010) Performance of the Cockcroft-Gault, MDRD, and new CKD-EPI formulas in relation to GFR, age, and body size. Clin J Am Soc Nephrol 5(6):1003-1009 